\title{
Handover Performance Evaluation and Discussion of Mobile IPv6 HUANG Xiuwen ${ }^{1, a}$ \\ ${ }^{1}$ Modern Education Centre, Wuhan Textile University, Wuhan, 430074,China \\ aemail: 574208769@qq.com
}

Keywords: Mobility protocol; Quality of Service; Handover; Performance Evaluation

\begin{abstract}
Future internet works will include large numbers of portable devices moving among small, wireless cells. Users demand seamless mobility and Quality-of-Service (QoS) provisioning to support real-time applications. Hierarchical Mobile IPv6 (HMIPv6) is designed to reduce the amount of signaling required and to improve handover speed for mobile connections. In this paper, the architecture and operation of Mobile IPv6 protocols is studied and evaluated based on the QoS parameter. The simulation was carried out by using the Network Simulator-2. The simulation results show that, HMIPv6 performs best compared to MIPv6. The MIPv6 suffers large handover latency.
\end{abstract}

\section{Introduction}

Next generation wireless networks offer the promise of high speed access as well as IP-based data services to the mobile hosts. Protocols would be required to maintain the same level of performance in the wireless networking environment with frequent handoffs, as in the wire-lined environment.

As a mobile node $(\mathrm{MN})$ travels between wireless cells, data transfer between the $\mathrm{MN}$ and the correspondent node $(\mathrm{CN})$ will be typically changed from an old to a new access router (AR). In most mobility solutions, this process involves changes of routing entries in the MN and the CN, in addition to some designated mobility agents (home agent and/or foreign agent), and is called a handoff. It must ensure that end-to-end connectivity is maintained in a seamless way despite the changed path. So, the most famous protocol that supports mobility in IP networks.

Mobile IPv6 (MIPv6) [12] describes how mobile node can change its point of attachment from one access router to another. Hierarchical Mobile IPv6 (HMIPv6) [11] is one of protocols that is designed to reduce the amount of signaling required and to improve handover speed for mobile connections.

In this paper, The architecture and operation of each protocol is studied and are evaluated based on the Quality of Service (QoS) parameter: handover latency. The simulation was carried out by using the Network Simulator-2.

The rest of this paper is organized as follows. Section II discusses Mobile IPv6 Handover Process. Section III presents our performance analysis. Finally, in Section IV, we conclude this paper and give the future work.

\section{Mobile IPv6 Handover Process}

The system for handoff is based on the following scenario: the MN has a regional Care-of Address RCoA1 and an on-link Care-of Address LCoA3, which is depicted in Fig.1. When the CN sends packets to the MN, the packets will be sent through MAP1 to the MN's LCoA3.

1. When the MN is about to move from the MAP1 domain to the MAP2 domain:

1) The MN sends a request control message to MAP1 to construct a multicast group for the MN.

2) MAP1 forms a multicast group for the $\mathrm{MN}$ and sends a multicast group join request to all other neighboring ARs. The neighboring ARs send response messages after receiving these multicast group requests toward MAP1 to show their availability to receive multicast packets from MAP1. 
3) The packets encapsulated by MAP1 are tunneled from the $\mathrm{CN}$ to the multicast group members. These ARs buffer the packets. As a final point, these neighboring ARs forward the packets.

2. When the MN travels from MAP1 domain to MAP2 domain:

1) The MN initially acquires a new address from the MAP2 network (RCoA2, LCoA4). The MN sends a Binding Update to MAP2 through AR4 and sends a message requesting AR4 to forward a multicast message. AR4 receives the request message, and subsequently forwards the buffered packets to the MN.

2) Whereas AR4 constantly sends multicast packets to MN, MAP2 receives the Binding Update and checks for DAD. MAP2 sends a Binding Update to the MN' s Home Agent after receiving the DAD. After that MAP2 waits for a binding acknowledgment from the Home Agent. MAP2 followed by sends a Binding Acknowledgment to the MN.

3) The MN receives the Binding Acknowledgment and sends a Binding Update to the CN via MAP2.

4) After receiving the Binding Update, $\mathrm{CN}$ changes the destination address RCoA1 to new RCoA2 and consequently directs the packets to MN in the new network via MAP2 and AR4.

5) AR4 stops sending multicast packets from MAP1 as soon as it receives new packets intended to the MN. MN at this moment receives packets directly from the $\mathrm{CN}$ as with Hierarchical Mobile IPv6.

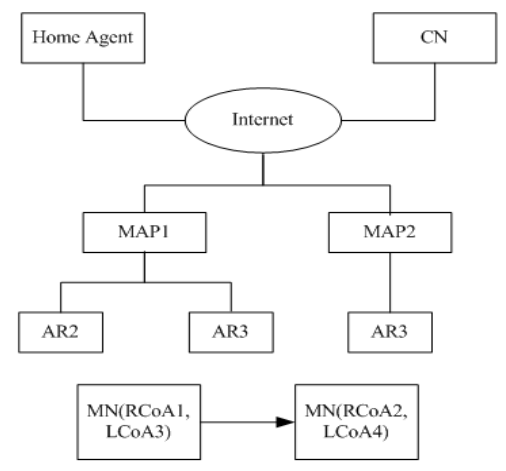

Fig.1. Inter-domain Handoff in HMIPv6

\section{Simulation And Discussion}

The Network Simulator, NS-2 [7] that supports for HMIPv6 which is ns-2.1b7a, was used for the evaluation of the protocols. The goal of this simulation is to examine and compare between HMIPv6 and MIPv6 in terms of handover latency. Handover Latency is defined for a receiving MN as the time that elapses between the last packets received via the old access router (oldAR) and the arrival of the first packet along the new access router (newAR) after a handover. Fig.2 shows the network topology used for simulation experiment handover.

The link characteristics namely the bandwidth (megabits/s) and the delay (milliseconds), are shown beside the link. The access routers are set to be 70 meters apart with free space in between. The wireless coverage area of the access router is approximately 40 meters. Finally, our model assumes a well-behaved mobile node movement pattern where the mobile node moves linearly from one access router to another at a constant speed of $1 \mathrm{~m} / \mathrm{s}$. Fig.3 explains the nodes topology for both protocols. 


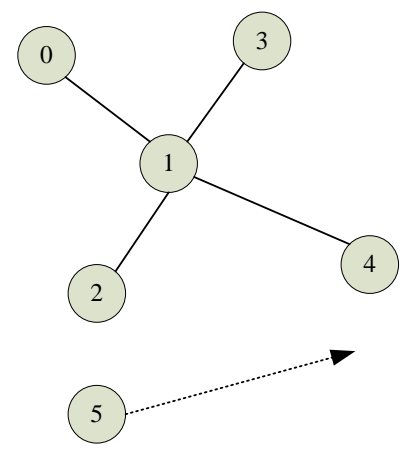

Fig.2. Simulation Network Topology

\begin{tabular}{|c|l|l|}
\hline \multirow{2}{*}{ Node } & Macro-mobility Protocol \\
\cline { 2 - 3 } & HMIPv6 Anchor & MIPv6 \\
\hline Node 1 & $\begin{array}{l}\text { Mobility } \\
\text { Point }\end{array}$ & - \\
\hline Node 2 & Old Access Router & Old Access Router \\
\hline Node 3 & Home Agent & Home Agent \\
\hline Node 4 & New Access Router & New Access Router \\
\hline Node 5 & Mobile Node & Mobile Node \\
\hline
\end{tabular}

Fig.3. Mode Description

Fig. 4 shows the handover effect where it is evaluated based on the graph of cumulative sum of the packets sent from $\mathrm{CN}$ to $\mathrm{MN}$ versus time in seconds.

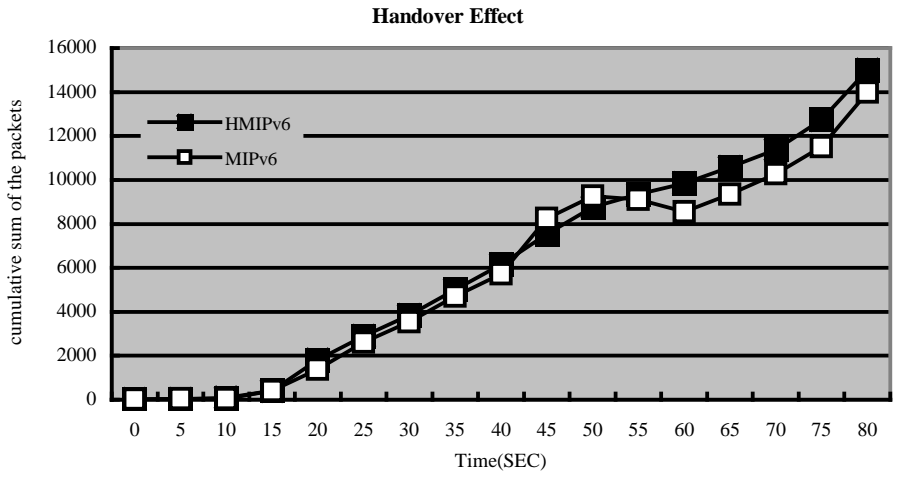

Fig.4. Handover latency in Mobile IPv6

The following observations can be made about Fig.4:

1) $\mathrm{CN}$ starts to communicate with $\mathrm{MN}$ by sending packets at $11 \mathrm{~s}$ after it finishes its registration and all the setup links with HA and MN.

2) Then, at around 40s, packets lost/reordering begin to occur where at this moment, MN has moved to a new AR. However, this situation only happened in HMIPv6. In MIPv6, even though it suppose to start at the same time but due to the location of HA is quite further away from MN, thus, the delay will be increased. Since MN is always contact to HA in order to tunnel the packet from/to $\mathrm{CN}$, then, it affects the movement of MN to the new AR. As a result of the packets lost/reordering, slow start activity can be observed thereafter.

3) After around t =45s for HMIPv6 and 49s for MIPv6, eventually, the transmission returns to normal. The overall handover latency, defined as the time when the MN detaches from the network at layer-2 till the disrupted communication session is returned to full operational state, is approximately 4500ms for HMIPv6 and 9000ms for MIPv6.

From the figure, we found that the time in HMIPv6 protocols between the last moment where the MN can receive and send packets through the old Access Router and the first moment where it can receive and send packets through the new Access Router is shorter compared to MIPv6.

\section{Conclusion}

Mobile IPv6 is a key element of the future of All-IP wireless network to allow users to traverse freely between domains and still be connected to a service network. This paper presents a comparative study between HMIPv6 and MIPv6. We have shown through the simulation that HMIPv6 perform best in terms handover latency compared to MIPv6. MIPv6 suffers longer handover latency because the time to send back the BU at new AR to the $\mathrm{CN}$ takes longer time. However, the handover latency for HMIPv6 is still quite large. Future work will have to design some scheme for reducing handover delay and packet loss in hierarchical Mobile IPv6 Networks. 


\section{Acknowledgment}

The authors wish to thank Professor Nie.Gang and Dr. Wu.Xinyi from Wuhan Textile University, China. This work was supported in part by a grant from Ministry of Education, Hubei province, China.

\section{References}

[1]Joe. Inwhee, Lee. Hyojin. An efficient inter-domain handover scheme with minimized latency for PMIPv6, International Conference on Computing, Networking and Communications (ICNC), pp. 332-336, March 2012.

[2]Sivakami.T., Shanmugavel.S.. An overview of mobility management and integration methods for heterogeneous networks, Third International Conference on Advanced Computing (ICoAC), pp.41-45, Dec. 2011.

[3]Bo Hu, Shanzhi Chen, Xiaoyan Jiang. A Performance Evaluation of IP Mobility Support Protocols, Second International Conference on Multimedia and Information Technology (MMIT), pp.17-20, April 2010.

[4]Xinyi Wu, Gang Nie. Comparative Study and Performance Analysis of the Macro-mobility Protocol, Asia-Pacific Conference on Information Processing (APCIP), pp.497-500, July 2009.

[5]Gang Nie, Xiuhua Qing. Analysis and evaluation of an enhanced handover scheme in hierarchical mobile IPv6 networks, International Colloquium on Computing, Communication, Control, and Management (CCCM), pp.329-332, Aug. 2009.

[6]Xinyi Wu, Gang Nie. Comparison of different mobility management schemes for reducing handover latency in Mobile IPv6, International Conference on Industrial Mechatronics and Automation(ICIMA), pp.256-259, May 2009.

[7]L. Chen, X. Cai, R. Sofia, and Z. Huang. A cross-layer fast handover scheme for mobile WiMAX, Proc. of Vehicular Technology Conference, Sep. 2007.

[8]H.-J. Jang, Y.-H. Han, and S.-H. Hwang. A cross-layering handover scheme for IPv6 mobile station over WiBro networks, Journal of KIISE, vol. 34, no. 1, Feb. 2007.

[9]T. Mahmoodi, V. Friderikos, O. Holland, and A. H. Aghvami. Cross-layer design to improve wireless TCP performance with link-layer adaptation, Proc. of Vehicular Technology Conference, Sep. 2007.

[10]H.S. Flarion, C.Castellucia, K. El-Malki, and L. Bellver. Hierarchical Mobile IPv6 mobility management (HMIPv6), IETF RFC 4140, August 2005.

[11]R. Koodly. Fast Handovers for Mobile IPv6 (FMIPv6), IETF RFC 4068, July 2005.

[12]D. Johnson, C. Perkins and J. Arkko. Mobility Support in IPv6, RFC 3775, June 2004.

[13]Andrew T. Campbell et al., Comparison of IP Micro-mobility Protocols, IEEE Wireless Communications Magazine, Vol. 9, February 2002.

[14]Xavier P' erez-Costa and Hannes Hartenstein. A Simulation Study on the Performance of Mobile IPv6 in a WLAN-Based Cellular Network, Computer Networks, special issue on "Towards a New Internet Architecture”, September 2002.

[15]Xavier P' erez-Costa and Marc Torrent-Moreno. A Performance Study of Hierarchical Mobile IPv6 from a System Perspective, Proceedings of IEEE International Conference on Communications (ICC), May 2003. 